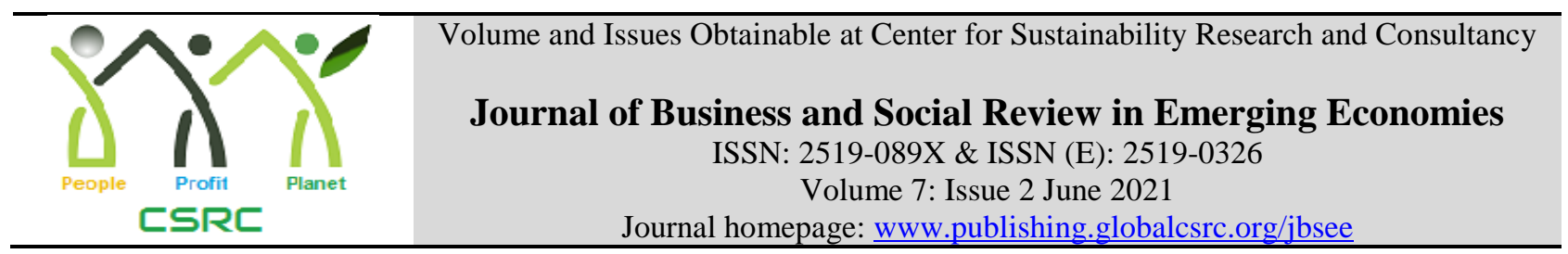

\title{
Speech-Language Intervention used by Professionals for Children with Autism Spectrum Disorder in Pakistan
}

\author{
*Shaheen Pasha, Department of Special Education (DOE), University of Education, Lahore, \\ Pakistan \\ Shakeela Shah, Dept. of Curriculum Development \& Instructions, Faculty of Education, \\ University of Sindh, Jamshoro, Pakistan \\ Ghazal Khalid Siddiqui, Department of Education, University of Education, Lahore, Pakistan
}

*Corresponding author's email: drshaheenpasha@hotmail.com

\begin{tabular}{l} 
ARTICLE DETAILS \\
\hline History \\
Revised format: May 2021 \\
Available Online: Jun 2021
\end{tabular}

\section{Keywords}

Speech-Language, Intervention, Autism, Speech therapy, and PECS

\section{JEL Classification} H75, I2O

\begin{abstract}
Purpose: This study will benefit the field of Autism as well as incorporation of communication interventions for the Professionals for Children with Autism in Pakistan. The objective of the study was to find the most common types of SpeechLanguage interventions used by professionals, Speech-Language interventions preferred by professionals, Speech-Language interventions supported by parents of children with autism.

Methodology: The study was quantitative the data was collected from the 100 health Professionals working in government and private autism centers or settings in different cities of Punjab. A purposive sampling technique was used. The researcher developed a questionnaire was developed by research considering literature review. The questioner had 35 questions related to interventions given by health professionals working in government and private autism centers.

Findings: Findings of the study showed that many institutions were giving various therapeutic services to children with Autism Spectrum Disorder regarding speech and language but mostly experienced people were practicing speech therapy and PECS as speech and language intervention.
\end{abstract}

(C) 2021 The authors, under a Creative Commons AttributionNonCommercial 4.0

Recommended citation: Pasha, S., Shah, S. and Siddiqui, G. K. (2021). Speech-Language Intervention used by Professionals for Children with Autism Spectrum Disorder in Pakistan. Journal of Business and Social Review in Emerging Economies, 7 (2), 369-374.

\section{Introduction}

Autism was an early childhood neurodevelopmental condition that could be characterized fully based on specific gaps in the child's social and emotional development, communication, and language processing. Autism was a behavioral disorder with a variety of traits that led to a significant socializing shortfall, significant unusual behavior, and limited repeated actions and sensitivity. Autistic children from early infancy will face difficulties in day-to-day activities 
The disability had a diverse variety that ranged from one individual to the other. As a consequence of a broad spectrum disorder, several scholars and trials have developed the disorder, with several recent pieces of the study carried out on this particular side. Autism impacted a person across his life so early diagnostics is very important for early interventions and a better life for children with autism. Recent research analysis has found that, before 2018, one in 59 children has autism; the CDC reported Autism has risen by $10 \%$ and one out of five children had autism

ASD was a delay in responsive and verbal expression, and a majority of the population examined did not improve their communication to the extent that was the most essential prerequisite for development. These limitations and delays, along with the increasing number of the population diagnosed with ASD, have resulted in a significant and urgent need for the recognition of evidence-based practices, especially for those who were unable to communicate using traditional methods.

\section{Literature review}

The autism disorder was a diverse neurodevelopment disorder, it was scientifically characterized by lack of socialization skill absence of creative playing, expressive and receptive language, repeated behavioral and specific patterns, desires, and action. The key characteristics of autism were based on three pillars: Dysfunction in both the social and non-social situations can result in inadequate or improper socialization. Rigidity and perseverance involve both stereotypic actions, such as meaningless repeated actions and resistance to adapt(Calkin, 2020).

In children with autism, language loss normally occurred very shortly when the kids reached age two. According to studies from parents of autistic children, 25 percent began producing speech around 12 months and 18 months before they lost language. Many trials and studies have shown that language and speech disorders have had sufficient impact on other aspects of development to play a significant role in improving communication with ASD children by developing successful therapies(Mehlenbacher, 2017).

Researchers have shown that children with autism are also struggling in various ways, such as socializing, communications, and cognitive processing that cause hearing incentives to be attended, imitated, controlled, and understood. Children with autism required alternate means of communication such that the sensory prompt could be in the form of pictures, cards, symbols, and written sentences, both tangible and subjective examples of the real world(La Roche, Bush, \& D'Angelo, 2018).

The relative effectiveness of the Picture Exchange Communication System (PECS) vs. a speechgenerating instrument (SGD) was discussed as a single topic longitudinal study for the acquisition of skills to request three primary children with extreme autism. The results showed an improvement in stressful output for all participants through both intervention processes(Koegel, Bryan, Su, Vaidya, \& Camarata, 2020).

Therefore, the vibrant need for initial and functional communication instruction for children with ASD was created. It will be impossible to choose the most effective technique for a single ASD child between parents and the psychiatrist in an environment where multiple communication training interventions were possible. Children with ASD had numerous characteristics and issues so that it could be hard to say which intervention is better for a specific individual or related to diagnosis and which children would benefit from augmentative and alternative communication. As well as on the other hand responses of different ASD children remained different to interventions so it is very difficult to reach one approach of intervention decisions(Alzrayer, 
Muharib, \& Wood, 2020).

As the reactions of multiple ASD children have been different from treatments, one solution to intervention decision is extremely hard to come up with. Language instruction explored speech and comprehension difficulties and complications and aimed to enhance oral, nonverbal, and social communication. The key aim was to encourage individual cooperation in more appropriate and effective ways. There would be differences in communication and language disorders in one another, others would not be able to communicate, some would not speak, nor could not speak and understand the physical reaction and facial gestures as they engage with others(Doak, 2018).

A SLP pathologist evaluated the strengths and weaknesses of the individual's comprehension, then established specific objectives before $t$ began language-speaking therapy. The main objectives of the treatment were to enhance linguistic abilities, to acquire non-verbal skills such as signals or expressions, and to interact by alternative methods (e.g. pictures and technology)(HICHOUR, 2019).

There have been several ways to improve practical communication skills. This system (PECS) has been considered a special training and development method designed to circumvent speech problems. To teach functional speech to autistic children and associated intellectual disorders, the Picture Exchange Communication System (PECS) was recognized and accepted. Its conjectural foundations are based on the applied study of actions and alternative and increased connectivity(Vento-Wilson, 2019).

PECS was recognized as a curriculum for manual education for children about the use of a clinical and school communications method dependent on an exchange; it was the traditional choice for care with Nonverbal ASD children. This technique has many benefits compared to the techniques of imitation (both verbal and gestural) and symbolic collection. The device started by swapping the one basic symbol and then progressed gradually into the form of the phrase. The machine emphasized also that the request utility was established earlier than its ability to address fundamental questions or to make various observations(Kurniawan, 2018).

Although training in functional communication and speech production may be seen as the priority of the PECS programs, it was established that it was an extremely valuable advantage. Persons that were nonverbal or spoke small wanted a contact device they could quickly start to use. You may know about the picture exchange communications scheme whether you are in special education or have a child with an autism diagnosis (PECS)(Hu \& Lee, 2019).

It was accepted because of its evidence-based practice and because it succeeded when applied in accordance. For objects, behaviors, and other individuals to be sought, PECS offered a very valuable methodology and applied behavior analysis theory to include and encourage social interactions. PECS began with random requests and moved to answer asking such questions about things in the environment(Ferreira et al., 2017).

It was also important to consider that while PECS was originally designed for younger learners with autism but was eventually used with a wide range of learners of all ages with different cognitive, physical, and communication issues and difficulties(Raja, Saringat, Mustapha, \& Zainal, 2017).

Additional techniques, such as manual signs, voice generators, naturalistic language training, and linguistic modelization were contrasted with PECS intervention. Several trials have shown some tests that PECS works better than sign and symbol, and that there is still little consideration behind PECS processing interference for speed and verbal stimulation technics with time-delay. 
Additional experiments by Gregory have shown that the participants replied differently(Chua \& Poon, 2018). This study will benefit the field of Autism as well as the incorporation of therapeutic interventions used by Professionals for Children with Autism in Pakistan.

\section{Objective}

The objective of the study was

1. To find the most common types of Speech-Language interventions used by professional in children with autism

2. To find out Speech-Language interventions preferred by speech professionals, clinical psychologists, educationists for children with autism

3. To find out Speech-Language interventions supported by parents for children with autism

\section{Methodology}

The study was quantitative the data was collected from the100 health Professionals working in government and private autism centers or settings in different cities of Punjab. A purposive sampling technique was used. The researcher developed a questionnaire was developed by research considering literature review. The questioner had 35 questions related to interventions given by health professionals working in government and private autism centers.

\section{Results}

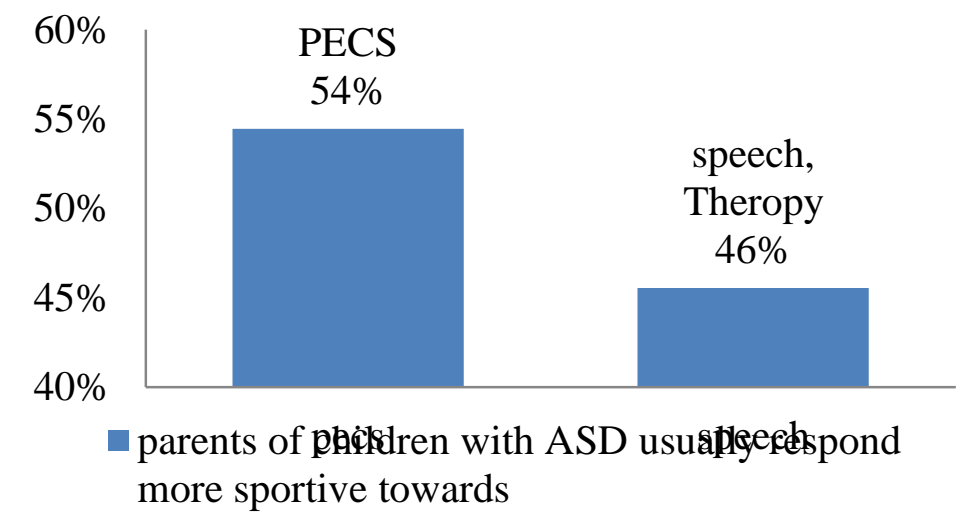

Fig: 1: Most common types of Speech-Language interventions used by professional in children with autism

The above figure showed that common types of Speech-Language interventions used by professional in children with autism were $\operatorname{PECS}(55 \%)$ while $45 \%$ was used speech therapy for improving communication in ASD children

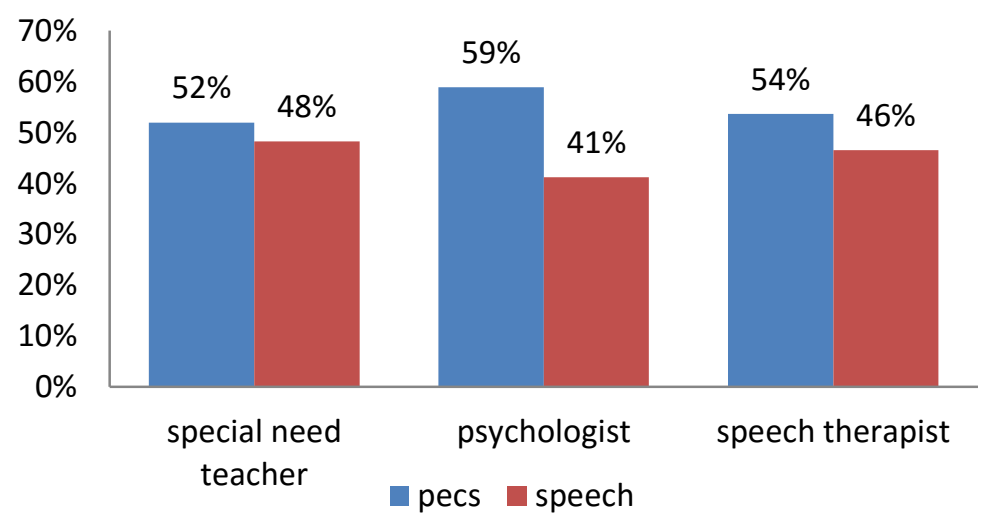


Fig 2: Speech-Language interventions preferred by speech professionals, clinical psychologists, educationists for children with autism

The above mention, figure shows that PECS was preferred by speech professionals, clinical psychologists, educationists for children with autism as compared to speech therapy

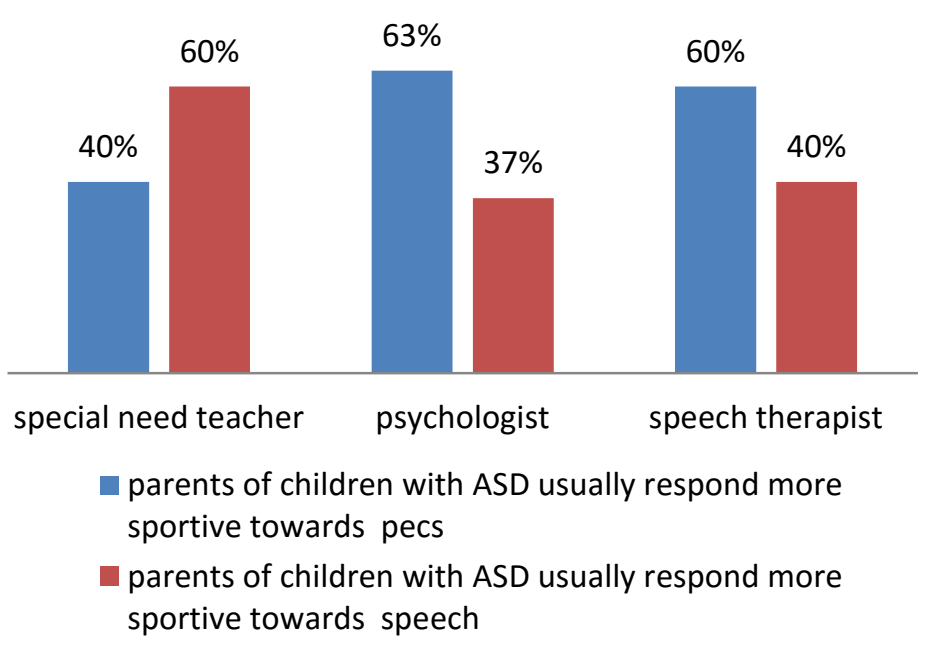

Fig: 3 Speech-Language interventions preferred by parents according to speech professionals, clinical psychologists, educationists for children with autism

The above figure Speech-Language interventions preferred by parents was PECS according to speech professionals, clinical psychologists, educationists for children with autism.

\section{Conclusion and Discussion}

The study found that many institutions offered different educational programs to children with autistic spectrum disorder of speech and language (speech, PECS interventions), but mainly experienced individuals who practiced speech and beginners became PECS followers. In any case, various measures are needed to overcome the special ability to communicate to children with an autism spectrum disorder.

Also, the analysis found that this trend had improved somewhat and that experts and clinicians did not reflect on alternative means of intervention with children with ASD with language learning. But in this sector, we also needed a huge area of transition. Also, in comparison with governmental institutions, our private institutions practiced the PECS program. Health professionals have contributed by suggesting that PECS activity played an important role in rapid language acquisition and communicating, but that the efficiency of speech operation remained lasting.

The study reveals, in comparison with speech therapies, that most psychologists practiced PECS for language learning in children with autism. On the other hand, second-number speech therapists preferred PECS intervention rather than speech and third-number special needs teachers favored PECS intervention. The study findings show that more parents of autistic children remain more content with PECS intervention than with speech therapies, according to Health Professionals.

\section{Recommendation}

The institutions should provide the parents of the children with an ASD with full knowledge of 
their communications issues with autism and provide appropriate advice or counseling on their therapy treatment through courses, seminars, and lectures. Institutions should schedule parents' meetings to raise awareness about concerns for other families with problems and parents to be involved in teachers' or institutions' meetings.

By giving relevant intervention, focus and events, professionals and parents alike will play a crucial role in developing childhood language and communication skills. Maximum educators and parents should be motivated to take part in these practices by communicative contact between infants, several websites and networks for children with ASD have been operating in this regard.

\section{References}

Alzrayer, N. M., Muharib, R., \& Wood, C. (2020). Effects of a behavior intervention package on augmented and vocal mands by children with developmental disabilities. Journal of Developmental and Physical Disabilities, 32(1), 57-74.

Calkin, B. (2020). Social Skills Interventions for Adolescents with Autism Spectrum Disorder in Ethnically Diverse Populations. Alliant International University.

Chua, B. Y., \& Poon, K. K. (2018). Studying the implementation of PECS in a naturalistic special education school setting. Educational and Child Psychology.

Doak, L. (2018). Exploring the multimodal communication and agency of children in an autism classroom. Sheffield Hallam University.

Ferreira, C., Bevilacqua, M., Ishihara, M., Fiori, A., Armonia, A., Perissinoto, J., \& Tamanaha, A. C. (2017). Selection of words for implementation of the Picture Exchange Communication System-PECS in non-verbal autistic children. Paper presented at the CoDAS.

HICHOUR, H. (2019). The Development of Pragmatic Communicative Skills in Speakers Children with Autism Spectrum Disorders: Case Study: Algerian Autistic Children.

$\mathrm{Hu}, \mathrm{X}$., \& Lee, G. (2019). Effects of PECS on the emergence of vocal mands and the reduction of aggressive behavior across settings for a child with autism. Behavioral Disorders, 44(4), 215-226.

Koegel, L. K., Bryan, K. M., Su, P. L., Vaidya, M., \& Camarata, S. (2020). Definitions of nonverbal and minimally verbal in research for autism: A systematic review of the literature. Journal of autism and developmental disorders, 1-16.

Kurniawan, I. (2018). The improvement of autism spectrum disorders on children communication ability with PECS method Multimedia Augmented Reality-Based. Paper presented at the Journal of Physics: Conference Series.

La Roche, M. J., Bush, H. H., \& D'Angelo, E. (2018). The assessment and treatment of autism spectrum disorder: A cultural examination. Practice Innovations, 3(2), 107.

Mehlenbacher, H. E. (2017). Group Versus Individual Intervention: A Best Practice Analysis for Social Skill Development in Children Aged Four to Seven with Autism Spectrum Disorder. City University of Seattle.

Raja, P., Saringat, M. Z., Mustapha, A., \& Zainal, A. (2017). Prospect: A picture exchange communication system (PECS)-based instant messaging application for autism spectrum condition. Paper presented at the IOP Conference Series: Materials Science and Engineering.

Vento-Wilson, M. (2019). The Intersection of Speech-Language Pathologists' Beliefs, Perceptions, and Practices and the Language Acquisition and Development of Emerging Aided Communicators. Chapman University. 